CBN96-16

\title{
Trapped Modes in CESR Sliding Joints
}

\author{
E. B. Anderson \\ J. Rogers \\ Department of Physics, Cornell University, Ithaca, NY
}

November 6, 1996

\begin{abstract}
Previous experiments had found apparent trapped modes in the sliding joints which would cause a longitudinal instability in the beam. Pairs of bunches were run at various spacings to attempt to detect the effects of the modes through wake field heating. Data taking was time consuming, and insufficient numbers of data points could be taken to fit to the wake fields. To help solve this, an attempt was made to measure the frequency of the modes on the bench. This attempt failed to find the previously observed modes and found indications that these were not trapped modes. A single trapped mode and its profile were observed. It was used to make predictions about the heating, shunt impedance, and longitudinal instability threshold due to the mode.
\end{abstract}

\section{Introduction}

Trapped modes in the sliding joints may eventually place a limit on the beam currents. Because of the sheer number of sliding joints in the ring, similar trapped modes in all of the joints could cause a significant instability in the beam in the future. This possibility has previously been investigated [1]. Further studies of this possibility were initiated by trying to measure the effect in the beam by using pairs of bunches at varying spacings [2]. The fields from the second bunch could strengthen or cancel the fields still remaining from the first bunch. It was hoped that this would change the heating caused by the second bunch in a noticeable way.

Searches for data to complement this information led to tests of a sliding joint using a network analyzer to search for the trapped modes. Attempts to locate the previously observed modes were unsuccessful and indications were found that these modes were not trapped modes. A single trapped mode was located just below the TM cut-off frequency. The profile of this mode was measured using a perturbative method. Using the profile and an approximate field configuration, the shunt impedance of the mode was estimated. These results were used in the program ZAP to look for possible instabilities. 


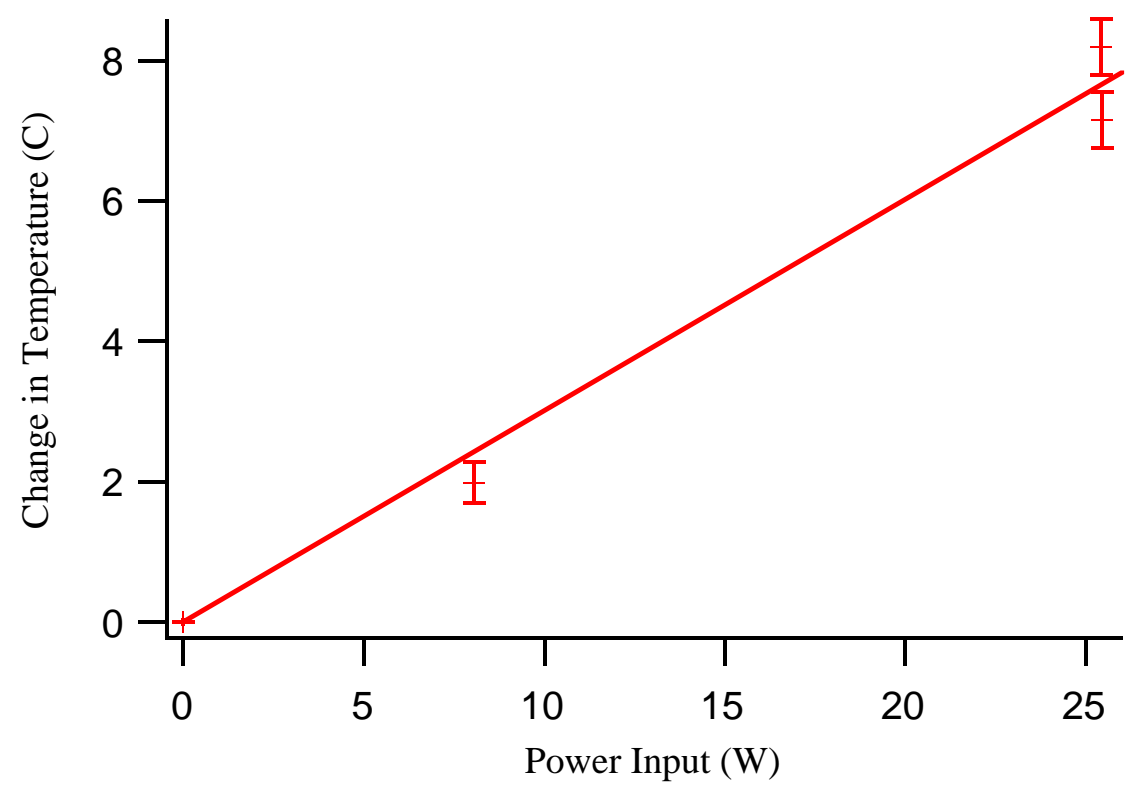

Figure 1: The plot shows the change in temperature versus energy input through resistors in the cooling channels.

\section{Two Bunch Tests}

In order to search for trapped modes in the sliding joints, a single beam, twobunch test was undertaken. The first bunch would excite any trapped modes. The second would pass through the fields. This would create varying amounts of heating depending on the phase of the trapped mode when the second bunch passed.

Various separations between the bunches were tested. Preliminary evidence suggested that the time constant for the heating of the sliding joints could be as high as ten minutes. Therefore, waiting for the temperature to stabilize was time consuming, and only a limited number of data points could be taken. Spacings varied from six to twenty-eight nanoseconds in two nanosecond steps.

Because the bunch measurements were made in terms of temperature, it was important to relate the temperature differences observed to amounts of energy lost in the sliding joint. The sliding joints were manufactured with a hole for a cooling pipe. This hole was not in use on most sliding joints at the time, and so it was a good candidate for heating the sliding joint externally. Resistors were lowered into these holes and thermally conductive grease placed around them. With no beam in the machine, known currents could be passed through the resistors at a known voltage. By relating the temperature gains to the energy lost through the resistors, an approximate calibration could be made. The data, shown in Figure 1, yields a temperature rise of approximately $3.3 \mathrm{C}$ per Watt 


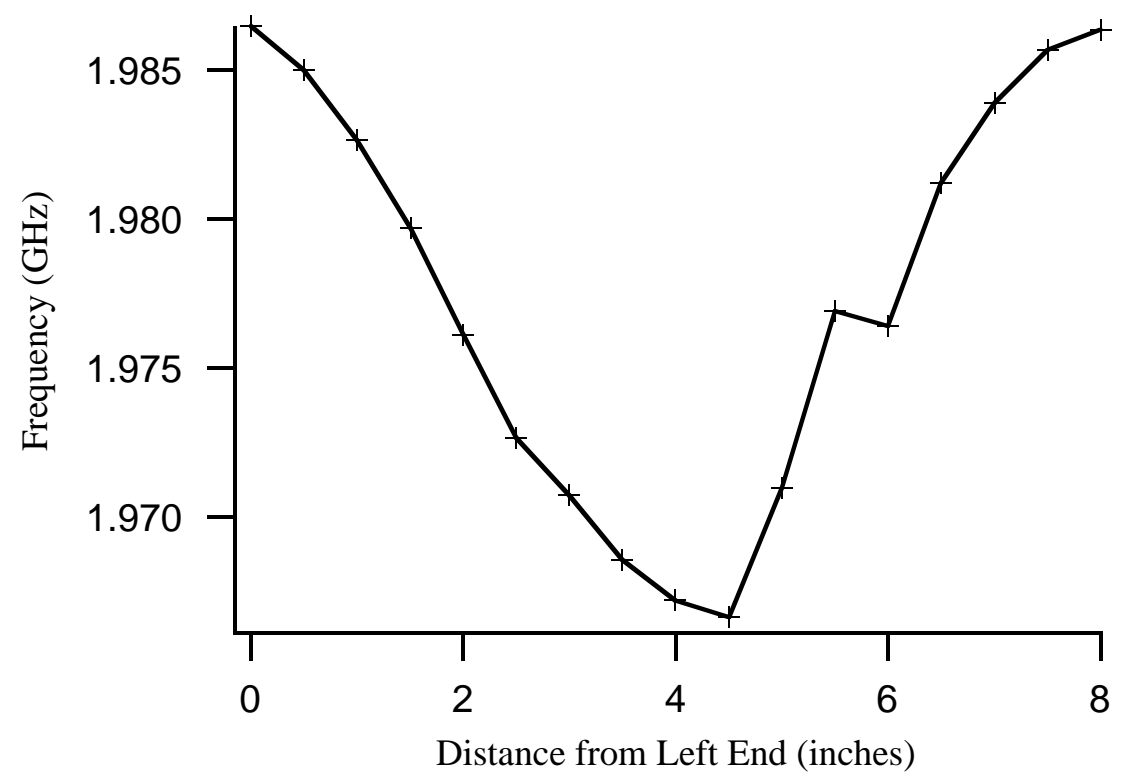

Figure 2: The peak at $1.98 \mathrm{GHz}$ shows a smooth profile with one minimum.

of power.

The data from the two bunch tests did not appear to represent a single mode in the joints, but there was insufficient data to fit two modes to the small number of data points. In order to fit this data, an attempt was made to measure the frequency of any trapped modes by making bench tests.

\section{Bench Tests}

In previous experiments [1], two apparently trapped $T M_{01}$ type modes were observed. These modes were noted at frequencies of 1.96 and $2.26 \mathrm{GHz}$ with nearly square longitudinal profiles of 14 and $8 \mathrm{~cm}$ respectively. An effort was made to measure these modes, but the $\mathrm{Q}$ values and profiles appeared to be quite different than were previously reported. The $\mathrm{Q}$ values for the modes were measured to be approximately 100 and 600 respectively, in contrast to the previously reported values of 2000 and 1000. A Slater perturbation method [3] was used to determine the field profiles of the modes. The clear peak at 1.98 $\mathrm{GHz}$ had a single minimum sinusoidal shape, while the peak at $2.32 \mathrm{GHz}$ had a two minima sinusoidal shape. There was a difficult to track peak at about $2.72 \mathrm{GHz}$, and a fourth peak with a sinusoidal field pattern with four minima at $3.28 \mathrm{GHz}$. Plots of frequency shift versus bead position for the modes can be found in Figures 2-4. This sequence of field profile minima suggests that these peaks are simply a series of standing wave modes inside of the cavity formed by the sliding joint and end plates. To test this assumption, a beam pipe 


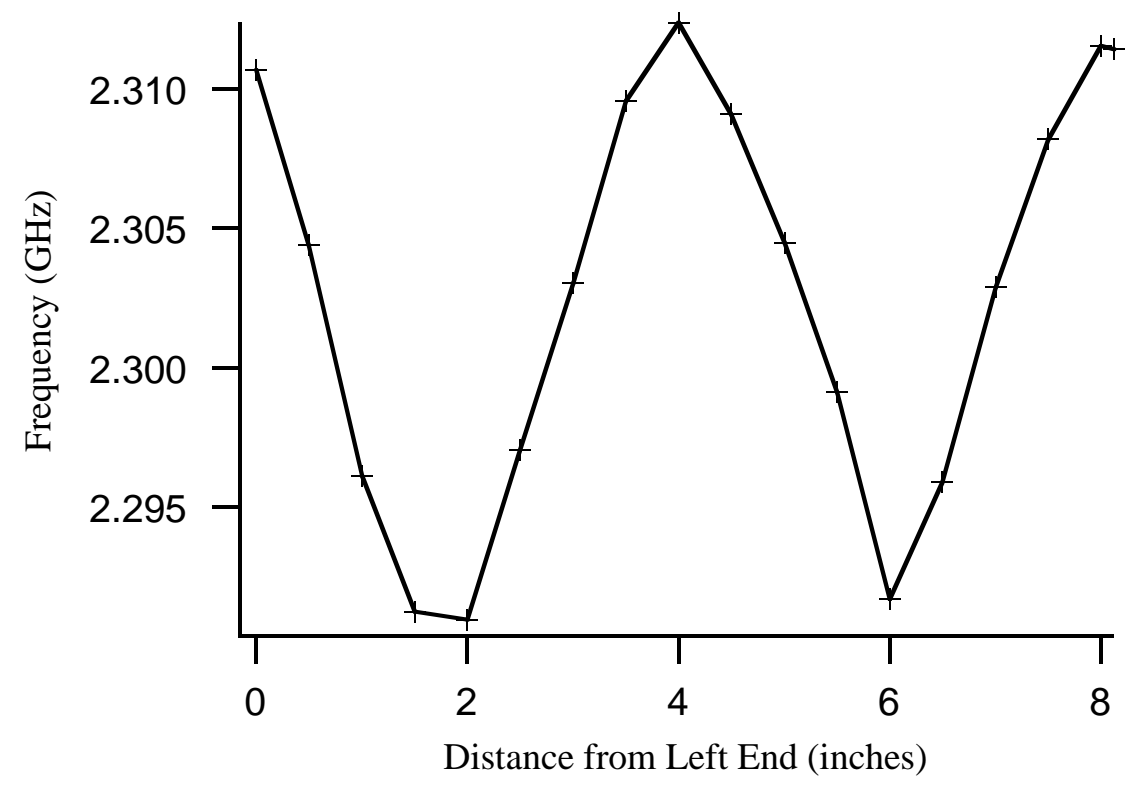

Figure 3: The peak at $2.32 \mathrm{GHz}$ shows a frequency variation with two minima.

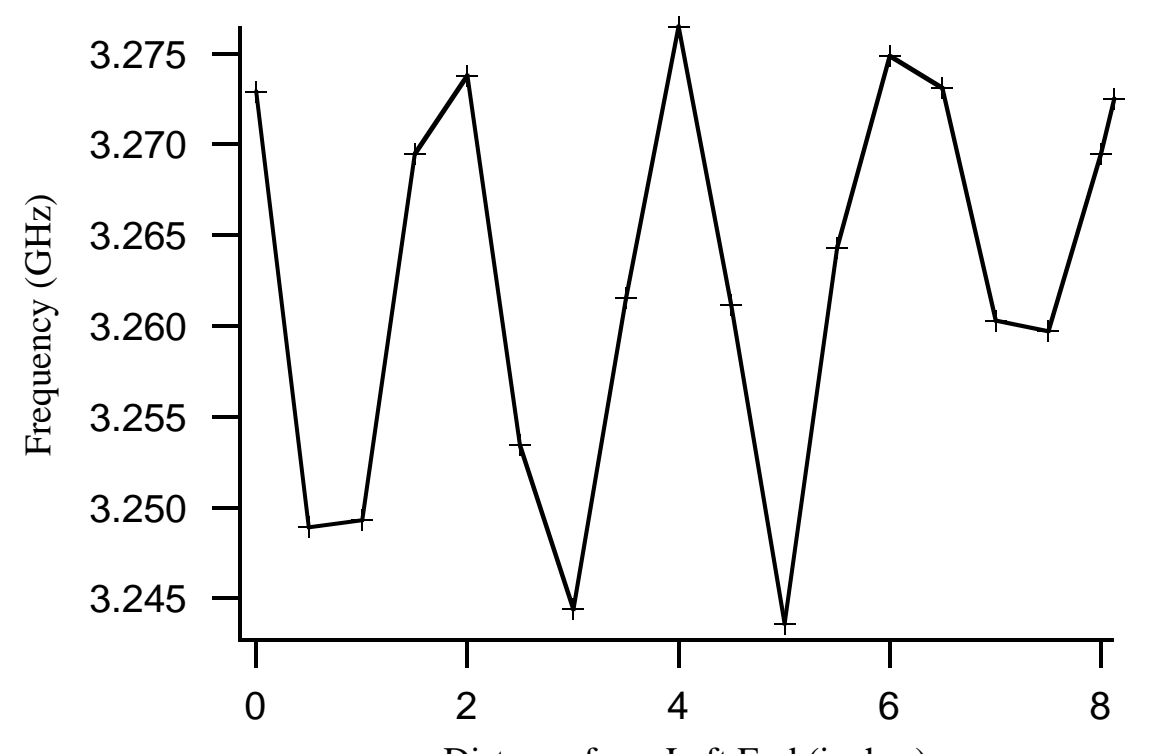

Distance from Left End (inches)

Figure 4: The peak at $3.28 \mathrm{GHz}$ shows a four minima smooth profile with one minima. 


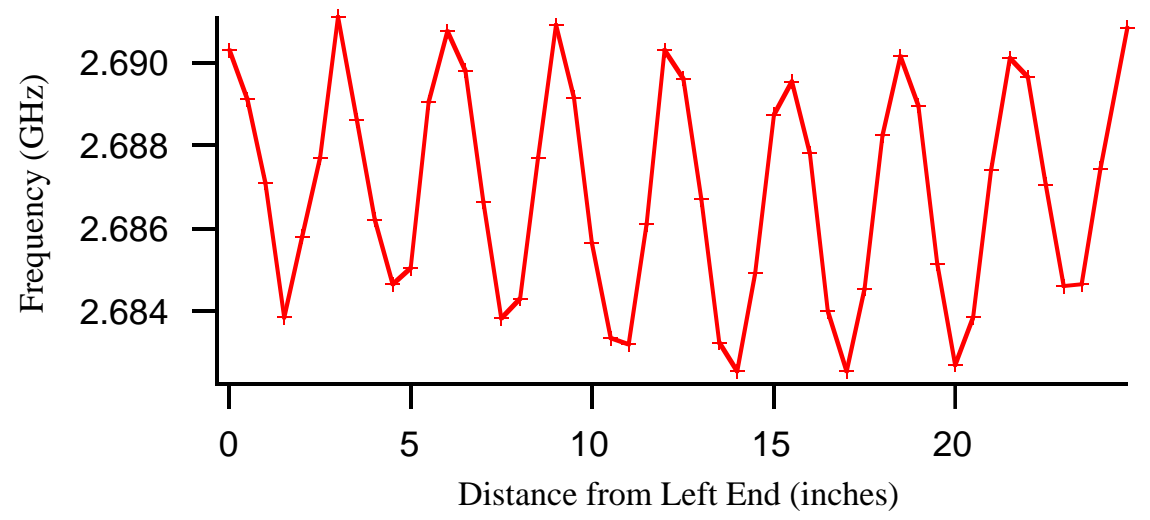

Figure 5: The peak at $2.69 \mathrm{GHz}$ shows a sinusoidal pattern and between the two end plates.

extension was added to one side of the sliding joint. This left a trio of peaks around $2.76 \mathrm{GHz}: 2.69 \mathrm{GHz}, 2.75 \mathrm{GHz}$, and $2.84 \mathrm{GHz}$. Investigation of the lowest peak showed a long sinusoidal field profile, as shown in Figure 5. The extended sinusoidal nature of this field profile encouraged further testing of end effects. Two sections of beam pipe were attached to the sliding joint with a series of beam ports along the upper surface so that probes could be lowered into the pipe at various locations. Probes were lowered into four different places along the beam pipe, and the various peaks noted compared to search for peaks that seemed independent of probe position.

In order to investigate only modes localized around the sliding joint, absorptive material was placed in the ends of the beam pipe sections. Two possible peaks were investigated: one at $1.73 \mathrm{GHz}$ and another at $3.70 \mathrm{GHZ}$. The field profiles, indicated by the frequency shifts in Figures 6 and 7, seems to indicate that these fields were associated with the areas where the beam pipe sections met the sliding joint. Because the sections of the apparatus were joined by tension rather than by welding, such a problem does not seem unusual.

The problem of differentiating cavity modes was addressed in the next measurement. It was important to be able to separate out the cavity modes, while also terminating the ends of the cavity to clean up the measurement. Sliding electrical shorts were constructed that could be moved freely along the inside of the beam pipe. Indium was used between the sliding joint and the beam pipe sections in order to improve the conduction at the joints. The length of the cavity could now be quickly and easily changed. Changing the length caused the cavity modes to move, but any trapped mode would maintain the same frequency. By using this method, an unshifting peak was spotted at 3.5977 GHZ, just below the TM cut-off frequency. In order to establish if this was a trapped mode, the profile was measured using a Slater perturbation method [3] identical to the one used above. The profile observed, shown in Figure 9, had the characteristic shape of a trapped mode located near the center of the 


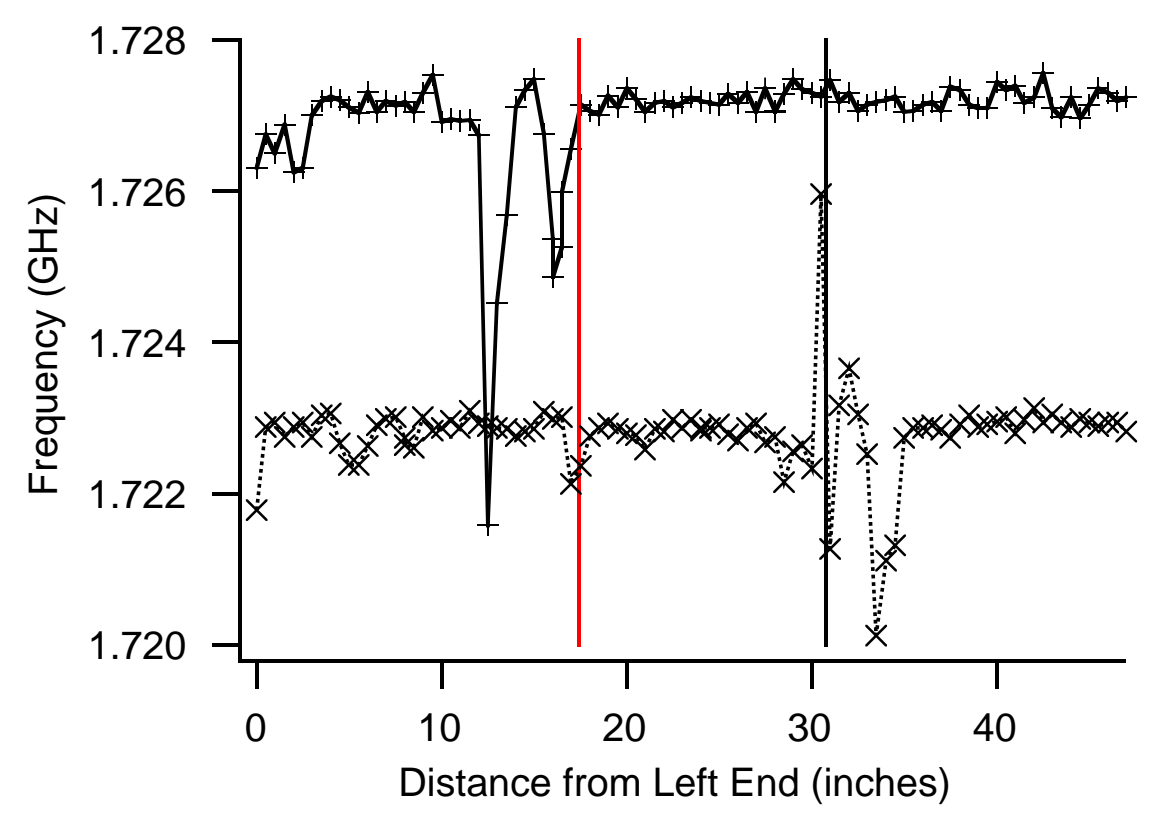

Figure 6: The peak at $1.73 \mathrm{GHz}$ shows a mode near the seam farthest from the damping material. The ends of the sliding joints are indicated by the vertical lines. 


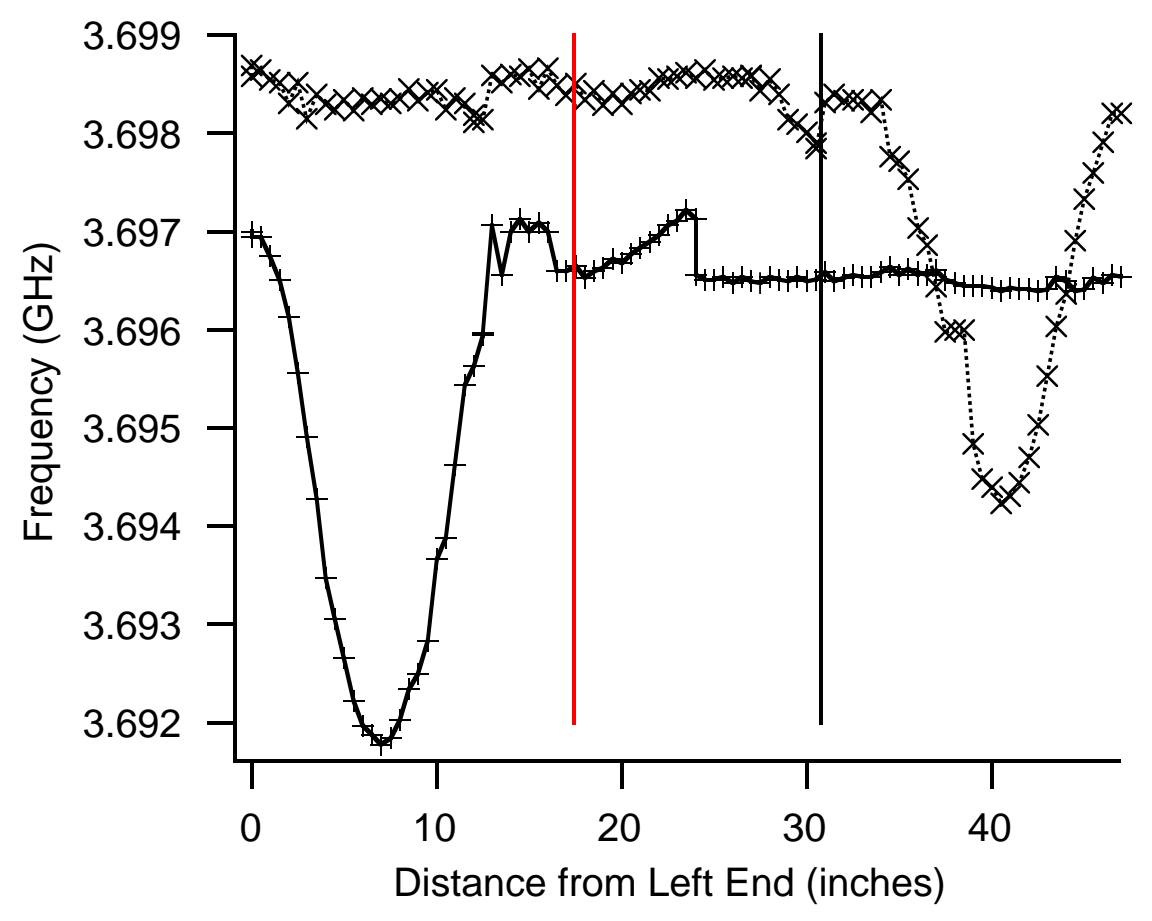

Figure 7: The peak at $3.70 \mathrm{GHz}$ shows a mode near the seam farthest from the damping material. The ends of the sliding joints are indicated by the vertical lines. 


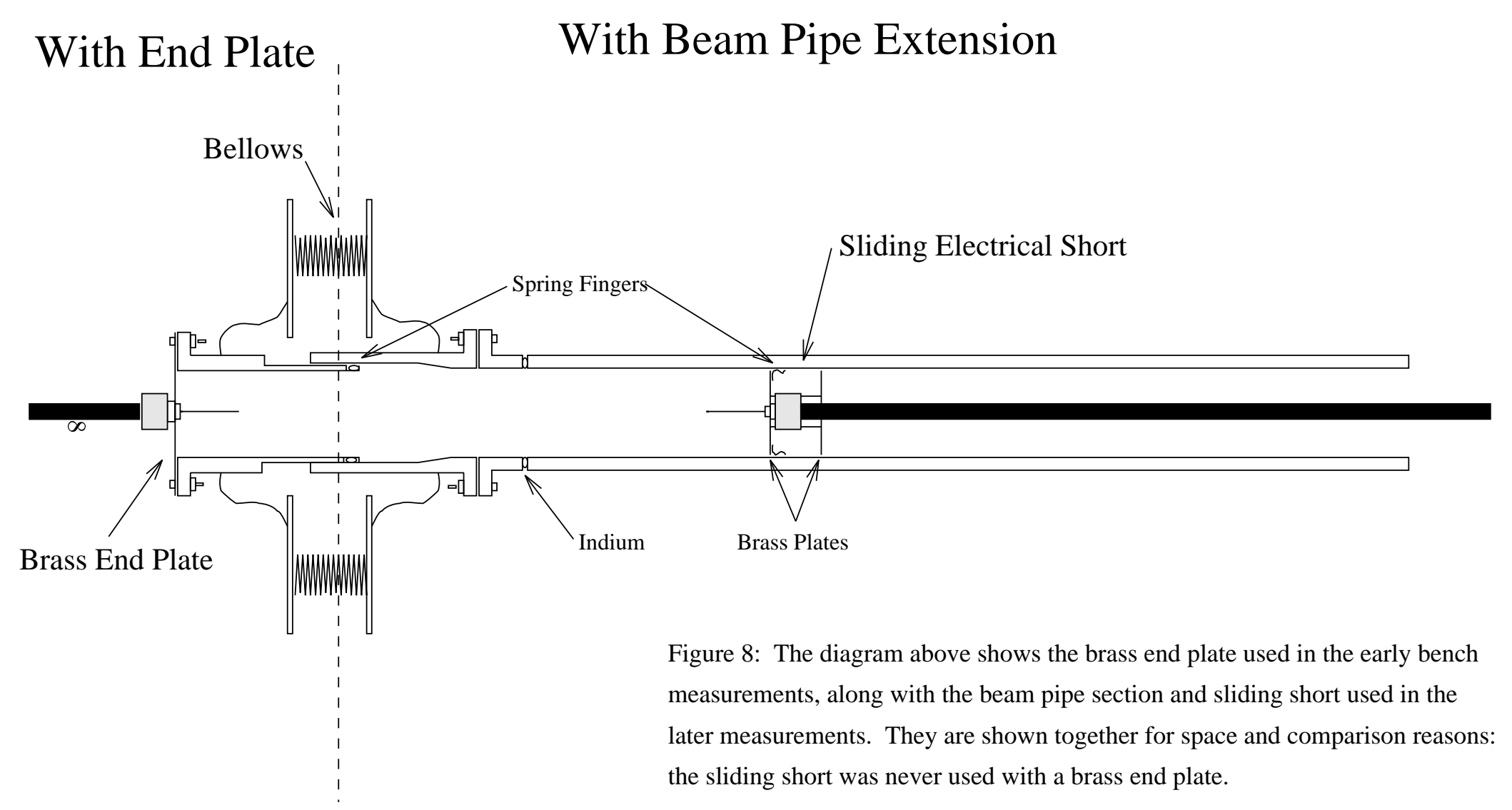




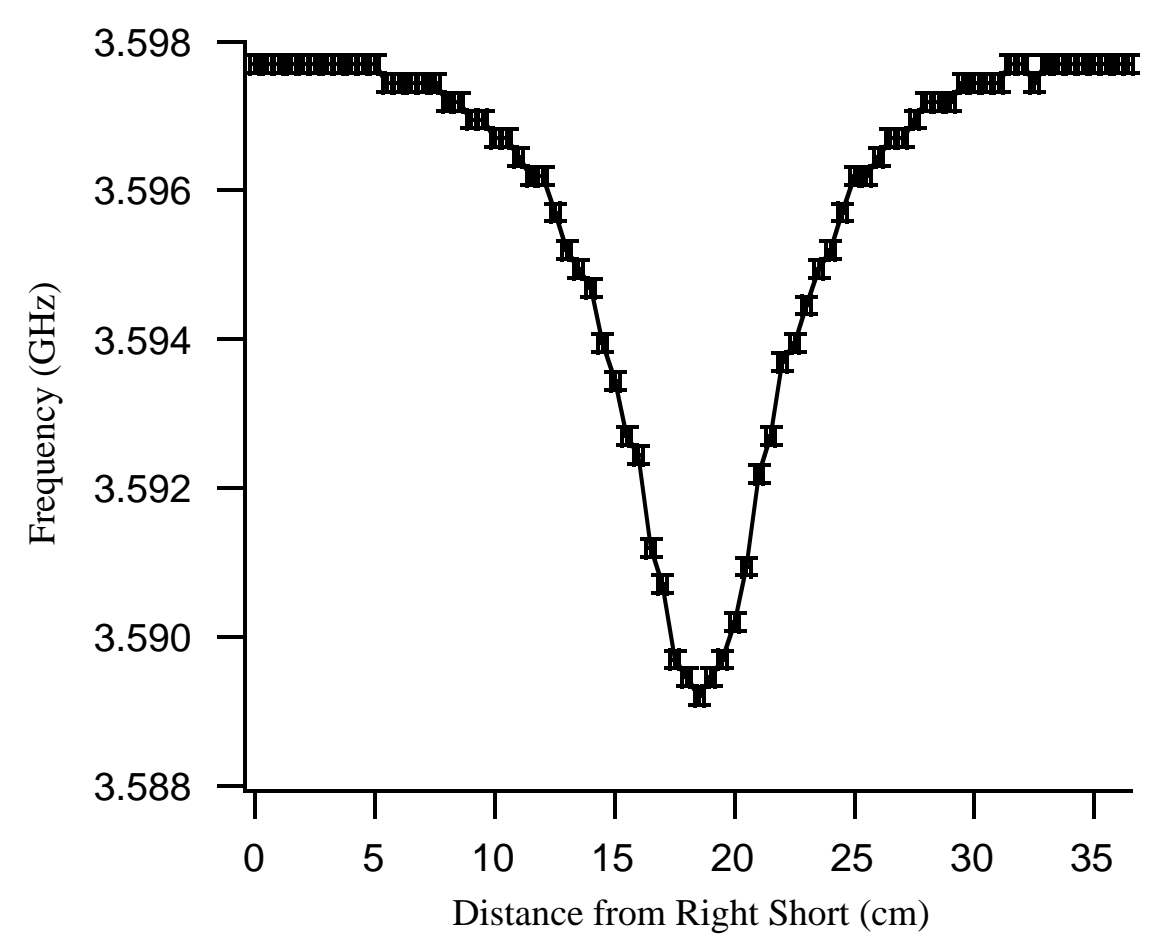

Figure 9: The plot above shows the change in frequency of the trapped mode as a function of the position of the perturbative bead. The change in frequency is approximately proportional to the change in the square of the field strength. 


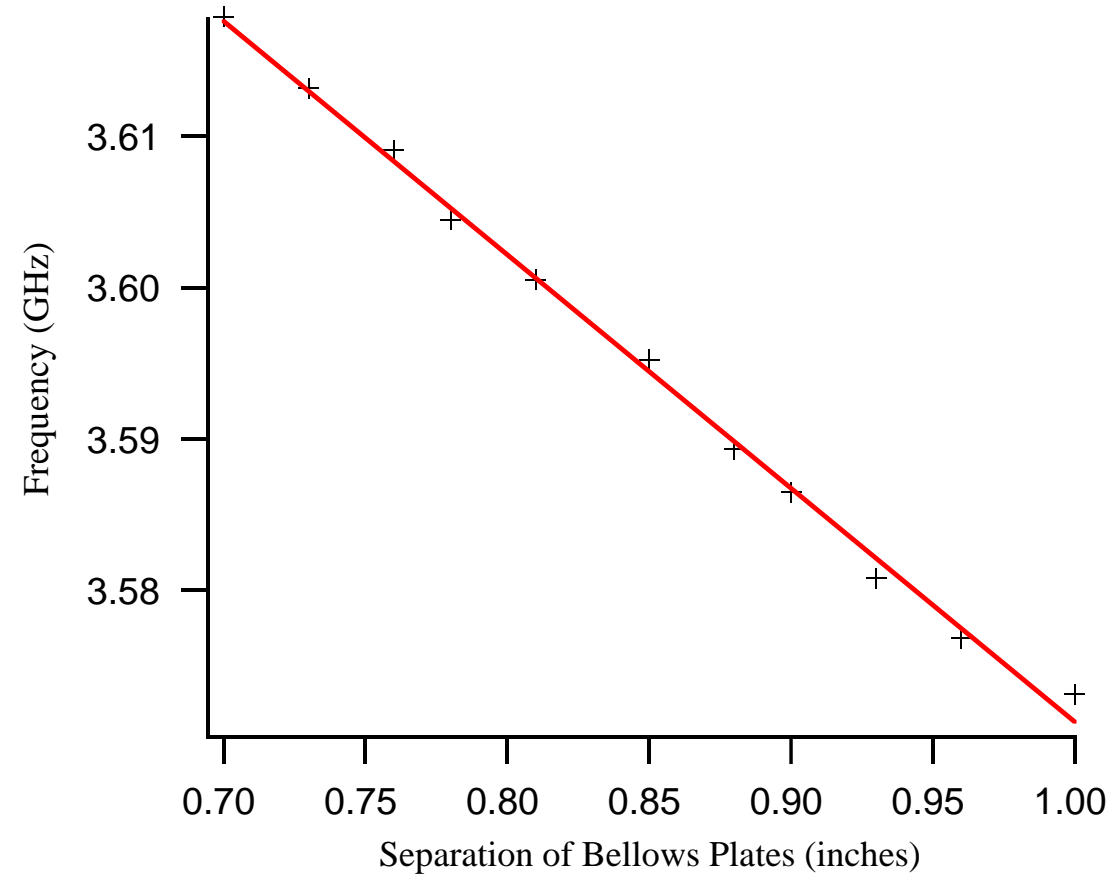

Figure 10: The frequency of the trapped mode changed as a function of the extension of the sliding joint. This is important since the extension of the joints vary greatly around the accelerator.

sliding joint. The $\mathrm{Q}$ value for the measure mode was at least 1800 , and seemed closer to 2000 when the contact between the beam pipe sections was particularly good. Because the actual extensions of the sliding joints in the accelerator varies appreciably, the frequency of the mode was measured as a function of the separation of the bellows plates. The original data was taken with a separation between the bellows plates of 0.83 inches. It can be seen in Figure 10 that the frequency changes over an approximately $50 \mathrm{MHz}$ range. Notice that the width of an individual peak is about $1.8 \mathrm{MHz}$, so the separations must be fairly close for the oscillators to interact.

\section{Calculation and Comparison}

Attempts were made to use the frequency measured above and make a fit to the two bunch test data. The calibration done above had already indicated that the temperature variations expected would be on the order of a degree. Attempts to fit to individual joints seemed dominated by noise, so an average was taken over all of the joints in the machine. There seemed to be a consistent change in temperature between data points. Unfortunately, the fits clearly indicated a 


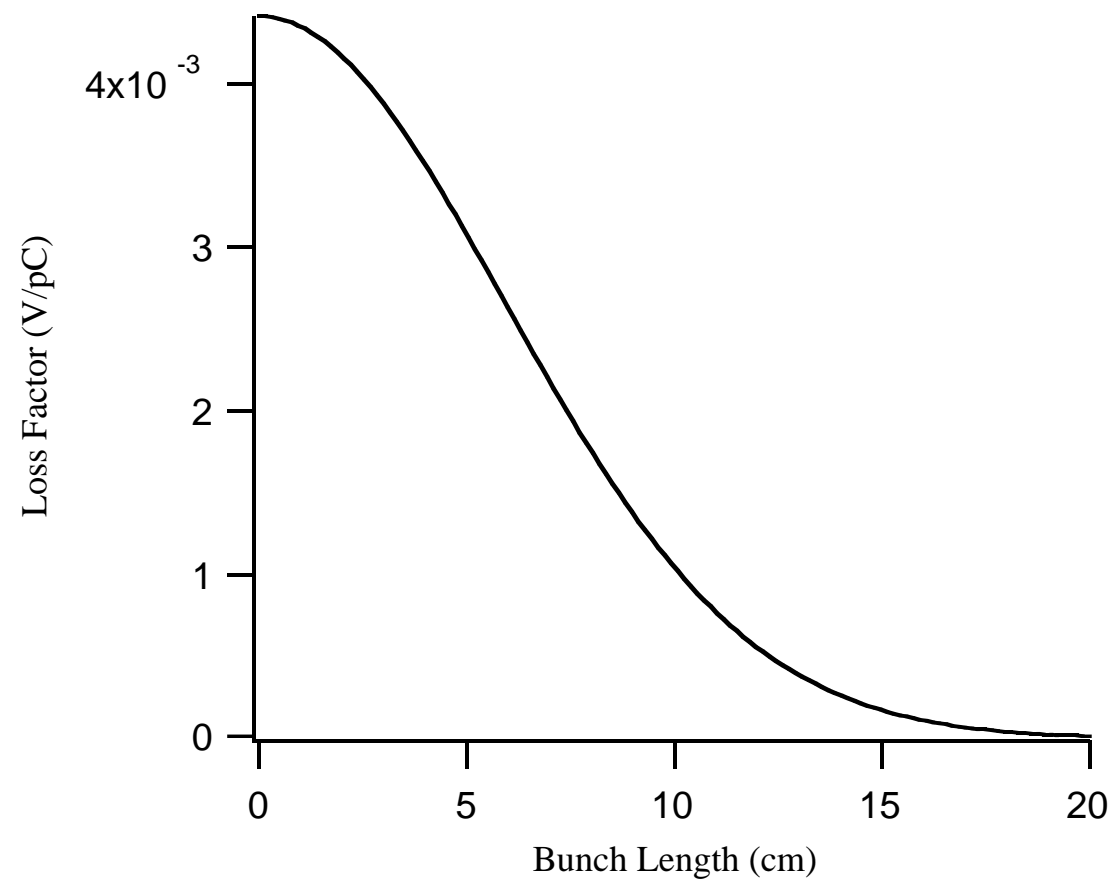

Figure 11: The loss factor due to the trapped mode should depend on bunch length in a Gaussian fashion, as shown above.

very non-physical result for the heating. When the fits were extrapolated to zero bunch spacing, the heating was not a maximum, in fact, it appeared to be near a minimum, which is clearly nonsensical. It seemed clear from the pattern of the points, and the non-physical nature of the fits that could be made, that current variation probably dominated any temperature effect that could be measured.

Knowledge of the relative field profile and the frequency of the mode allowed the calculation of the transit time factor for the mode. The value for this factor was 0.787 .

Previous bench measurements [4] had determined an approximate total loss factor for the sliding joint of $3.9 \times 10^{-3} \frac{V}{p C}$ at a bunch length of $1.8 \mathrm{~cm}$. The loss factor due to the trapped mode should depend on bunch length in a Gaussian fashion as shown in Figure 11. Information about the trapped mode was used to determine its contribution to the total loss factor of the sliding joint. The field configuration in the sliding joint was estimated by using the transverse dependence of the calculated fields of the lowest order TM mode [5] and the longitudinal profile measured above. This field configuration was used to calculate the stored energy in the mode and, thus, the shunt impedance of the mode. This calculation gave a shunt impedance $\left(\frac{R}{Q}\right)$ of 4.9 ohms. This impedance relates to a loss factor of $4.2 \times 10^{-3} \frac{V}{p C}$ for the trapped mode in the sliding joint at a bunch length of $1.8 \mathrm{~cm}$. Note that this value is higher than the measured 
Table 1: Accelerator Parameters Used in ZAP Simulations

\begin{tabular}{lc} 
Machine & $\begin{array}{c}\text { Value } \\
\text { Used }\end{array}$ \\
\hline Parameter & $766.63 \mathrm{~m}$ \\
Avercumference $\beta_{x}$ & $20 \mathrm{~m}$ \\
Average $\beta_{y}$ & $20 \mathrm{~m}$ \\
Effective Beam & \\
Pipe Radius & $2.5 \mathrm{~cm}$ \\
$\alpha$ & 0.011804 \\
Beam Energy & $5269 \mathrm{MeV}$ \\
$\sigma_{p}$ & 0.0061494 \\
Bunch Length & $1.8 \mathrm{~cm}$ \\
X Emittance & $7.64 \times 10^{-8}$ \\
Y Emittance & $2.55 \times 10^{-9}$ \\
Wall Material & $\mathrm{Al}$
\end{tabular}

value for the total loss factor, which includes all of the modes in the joint. In any case, the high value of the loss factor for the mode suggests that the trapped mode is the dominant source of impedance in the sliding joint.

Under normal operating conditions, the loss factor above relates to about $80 \mathrm{eV}$ lost per sliding joint per electron. Because there are one hundred sliding joints in the ring, the total energy loss would be $8 \mathrm{keV}$ per revolution.

The program ZAP [6] was now used to estimate the effects of the trapped mode on beam stability. The program could not deal with the large number of actual sliding joints in CESR. A few different ways to simulate them were tried. A single mode with one hundred times the impedance was tried first as a very rough measure. This rough test came up with all stable modes under normal operating currents, and an instability did not occur until a current of $2.13 \mathrm{~A}$ was used. Next, twenty sliding joints were used, with each having five times the impedance of a single joint. In this test, the there were some Landau damped modes over the entire reasonable current range. Unstable modes appeared at currents of $2.17 \mathrm{~A}$, a value well above current operating conditions.

For all tests where more than one mode was used, the frequencies of the sliding joints were not all assumed to be the same. The distribution of sliding joint extensions in the ring had been sampled previously [7]. Of the hundred sliding joints in the accelerator, the extensions of 31 were measured for the above paper. To determine the extension of a random joint, a measured value was selected at random. The extensions were measured to the nearest thirty second of an inch, but were only measured at one point around the rim of the bellows plates, so significant error was possible in just this measurement. A histogram of the expansions measured is given in Figure 12. To simulate these factors, a gaussian deviate of one thirty second of an inch was added to the randomly selected measured value. Using the measurement discussed above, 


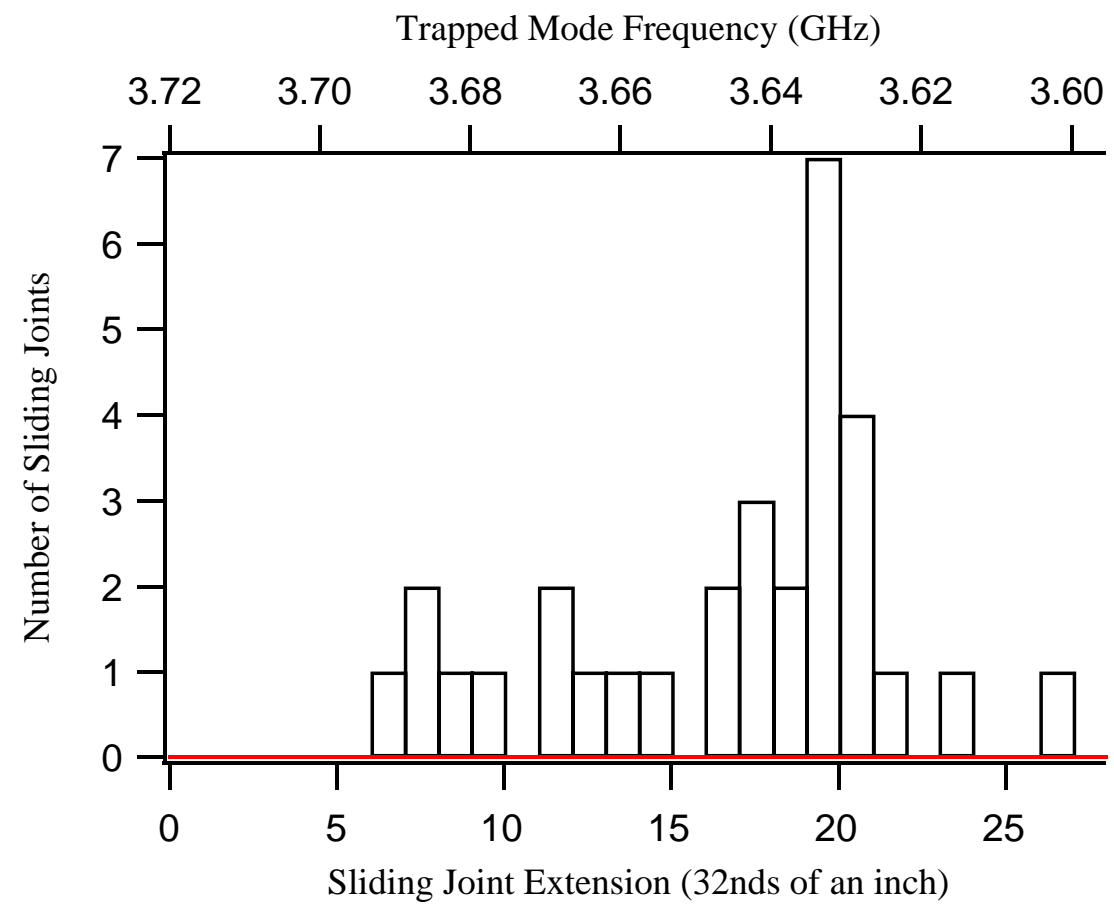

Figure 12: The various sliding joint expansions measured are given in the table above. Notice the variation in the frequency of the trapped mode over this range. 
the extension was translated into a frequency for the trapped mode.

\section{Conclusion}

Trapped modes in the sliding joints may eventually place a limit on the beam current. Machine studies generated interesting data, but data collection was too time consuming for the number of unknowns involved. Attempts to measure the frequencies using a network analyzer shed doubt on earlier measurements of the mode frequencies, but indicated a trapped mode in the sliding joint at about $3.60 \mathrm{GHz}$ with a $\mathrm{Q}$ factor of about 2000 . The mode observed predicted a loss factor of $4.2 \times 10^{-3} \frac{V}{p C}$ due to the trapped mode. This value is slightly higher than expected from earlier measurements, but indicates the importance of the trapped mode in the overall impedance of the structure.

\section{Acknowledgments}

Special thanks is given to Maury Tigner and Mike Billing for helpful discussion. Thank you to S. S. Kurennoy for his theoretical estimates. The bench studies were completed with the gracious loan of space, equipment, and time from the SRF group here at Cornell.

\section{References}

[1] Tigner, Murray. Trapped Modes in the CESR Sliding Joint and Collective Instabilities in PH II and III - a Progress Report, CON93-11.

[2] Temnykh, Alexander. Wake Field Study Using Two Spaced Bunches, CON95-06.

[3] Ginzton, Edward. Microwave Measurements, McGraw-Hill: New York (1957).

[4] Billing, Mike. Higher power Mode Loss Limitations for Beam Currents in CESR. CBN 84-15 (1984).

[5] Belomestnykh, Sergei. Personal Communication.

[6] Zisman, Michael, Swapan Chattopadhyay, and Joseph Bisognano. ZAP accelerator physics code.

[7] Tigner, Murray. Personal Communication. 